\title{
Lesion-Specific Coronary Artery Calcium Quantification for Predicting Cardiac Event with Multiple Instance Support Vector Machines
}

\author{
Qingshan Liu ${ }^{1}$, Zhen Qian ${ }^{2}$, Idean Marvasty ${ }^{2}$, Sarah Rinehart ${ }^{2}$, Szilard Voros ${ }^{2}$, \\ and Dimitris N. Metaxas ${ }^{1}$ \\ 1 Rutgers University, Piscataway, NJ, 08854 USA \\ 2 Piedmont Heart Institute, Atlanta, GA, 30309, USA
}

\begin{abstract}
Conventional whole-heart CAC quantification has been demonstrated to be insufficient in predicting coronary events, especially in accurately predicting near-term coronary events in high-risk adults 1 . In this paper, we propose a lesion-specific CAC quantification framework to improve CAC's near term predictive value in intermediate to high-risk populations with a novel multiple instance support vector machines (MISVM) approach. Our method works on data sets acquired with clinical imaging protocols on conventional CT scanners without modifying the CT hardware or updating the imaging protocol. The calcific lesions are quantified by geometric information, density, and some clinical measurements. A MISVM model is built to predict cardiac events, and moreover, to give a better insight of the characterization of vulnerable or culprit lesions in CAC. Experimental results on 31 patients showed significant improvement of the predictive value with the ROC analysis, the net reclassification improvement evaluation, and the leave-one-out validation against the conventional methods.
\end{abstract}

\section{Introduction}

Atherosclerosis is the leading cause of morbidity and mortality worldwide. Later stages of atherosclerosis are characterized by progressive deposition of calcium in the coronary arterial vessel-wall. CT-based CAC scanning is a three dimensional imaging technique that efficiently quantifies calcium in the coronary vasculature in a non-invasive, low-radiation way. CT-based CAC measurements are widely used clinically for cardiac disease diagnosis and treatment planning.

Presently the popular CAC measurements are the whole-heart Agatston score (AS) [2] and the whole-heart volume score (VS) [3]. The AS is calculated by multiplying the area of calcification by an arbitrary weighted density score based on the maximum Hounsfield unit (HU) value. The VS is defined as the total volume of the calcification in the major epicardial arteries, where calcification is identified as voxels with $\mathrm{HU} \geq 130$. The whole-heart AS/VS have been validated to be independent of, and additive to, the Framingham Risk Score (FRS) in predicting major cardiovascular events [4. Recently, a coronary calcium coverage score 
was proposed to quantify the percentage of coronary arteries affected by calcific plaque, but it was not superior to the whole-heart AS/VS in prediction of hard cardiac events 5. Some standard statistical methods, such as Cox regression and Kaplan-Meier estimator, are widely used to evaluate predictive values of coronary events on relatively long term data sets [6], and a very significant conclusion has been made: the absolute CAC measure is more predictive of coronary events than other regular clinical measurements. However, the information obtained by the relatively long-term studies is limited to individual patient treatment. In clinics, near-term event prediction is more desirable for treatment planning in intermediate to high-risk patients. In [1], a comparison study has shown that the whole heart AS/VS CAC measurements does not accurately predict near-term future coronary events in high-risk adults.

In this paper, we aim at improving CAC's near-term predicative value of major adverse cardiac events (MACE) including death, nonfatal myocardial infarction, and revascularization, in intermediate to high-risk population by using lesionspecific CAC quantification. Besides the whole heart AS/VS, a three dimensional CAC volume contains other clinically significant relevant information, such as the number of plaque lesions and geometric characteristics of lesions. These lesion-specific CAC evaluations will be more helpful for near-term prediction of MACE than the conventional whole heart CAC. For instance, the decision making of revascularization is largely based on the severity and location of the arterial stenosis. 8] showed that distance to corresponding ostium was a good indicator in analyzing cardiac events. However, different patients have different numbers of calcific lesions with different lesion distributions. MACE is generally caused or induced by one or a few culprit or vulnerable lesions, but it is hard to discriminate such risky lesions without further examinations. Thus, conventional pattern-level supervised learning approaches can not work well.

To handle these issues, we proposed to use multiple instance support vector machines (MISVM) 9] to estimate the predictive value of CAC scan for the incidences of MACE. The idea of MISVM is to perform a soft-margin based SVM on the unobserved instances, subjected to constrains defined by the labeled bags. The bag is labeled as positive if at least one instance in a bag is positive. Otherwise a bag is negative. We model each patient's lesion-specific CAC quantification results as a bag of instances, in which each lesion is taken as an instance. While each instance may posses an associated true label, i.e., leading to a event (positive) or not leading to a event (negative), the labels of the instances are only implicitly accessible through the labels associated with the bags. The MISVM model can not only predict the event risk efficiently, but also it can evaluate the culprit or vulnerable lesions. We assessed our method on 31 patients' data including 11 patients with MACE and 20 patients without MACE all within 2 years after the CAC scan. Experimental results showed it significantly improved the prediction accuracy with the ROC analysis, the net reclassification improvement evaluation, and the leave-one-out testing compared to the conventional methods including the whole AS/ VS [2]3] and FRS [4]. 


\section{Methodology}

\subsection{Lesion-Specific CAC Quantification}

Although the whole-heart $\mathrm{CAC}$ scores can indicate the risk of cardiac disease, the lesion-specific details of the calcification have a potential to play an additive and indispensable role in determining the extent and severity of the coronary disease. In this paper, we aim to extract lesion-specific detailed information for CAC event analysis. Our method works on data sets acquired with typical clinical imaging protocols on conventional CT scanners without modifying the CT hardware or updating the imaging protocol.

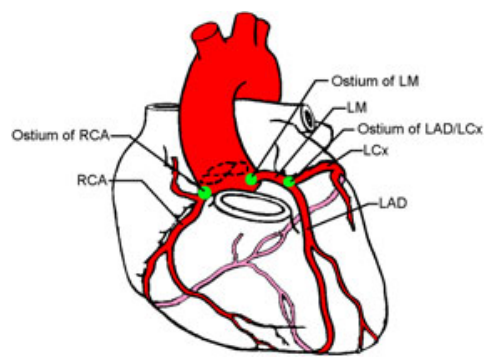

Fig. 1. The major coronary arteries and the arterial ostia

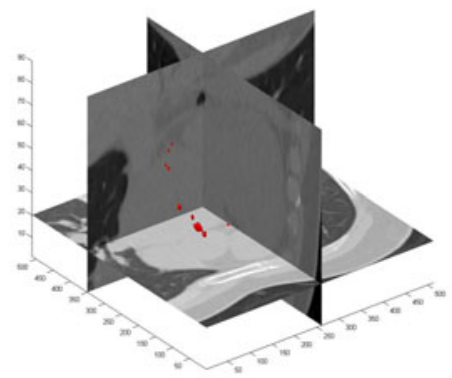

Fig. 2. Calcific lesion segmentation result

We only take account of 4 major coronary arteries: the left main coronary artery (LM), the left anterior descending artery (LAD), the left circumflex artery $(\mathrm{LCx})$ and the right coronary artery (RCA), as shown in Figure 1. A simple but efficient 3D 6-connectedness flood-fill operation is adopted to segment the lesions, which is defined as a group of calcific voxels that are 6-connected to each other, but not connected to any other calcific voxel in the binary calcium volume. The definition of 6-connectiveness is shown in Figure 3 (a) the blue voxel in the center is 6-connected to its 6 neighboring green voxels; (b) Voxel 1 is 6 -connected with voxel 2 by a 6 -connected path. Starting from the userspecified seed point in an epicardial arterial calcified lesion, the 3D lesion is easily segmented by propagating the 3D 6-connectedness flood-fill operations.Figure 2 shows the segmentation result of one sample. The locations of the major arterial ostia are identified manually in the 3D CAC volume.

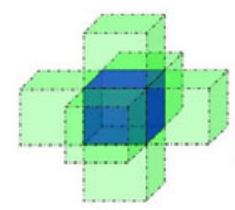

(a)

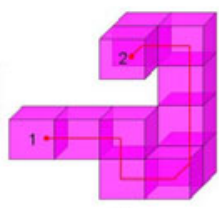

(b)

Fig. 3. 6-connectiveness in $3 \mathrm{D}$ volumes

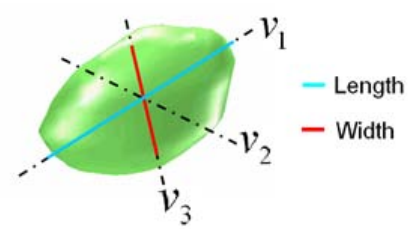

Fig. 4. The lesion's length and width 
Suppose lesion $\boldsymbol{L}$ has $N$ voxels with physical coordinates $p_{i}, i=1,2, \cdots N$ and the same resolution of $r=\left(r_{x}, r_{y}, r_{z}\right)^{\prime}$; The physical coordinates of the corresponding artery ostium is $p_{o}$. We quantify $\boldsymbol{L}$ with a 10 dimensional feature covering geometrical information, density, and clinical measurement as:

Length and Width: Given the covariance matrix of the voxels in the lesion, we calculate its eigenvectors $v_{1}, v_{2}, v_{3}$ and corresponding eigenvalues $\lambda_{1}, \lambda_{2}, \lambda_{3}$. Assuming $\lambda_{1} \geq \lambda_{2} \geq \lambda_{3}$, we define the length as the maximum distance between any two lesion voxels along the direction of $v_{1}$, and the width is the maximum distance between any two lesion voxels along the direction of $v_{3}$, $L=\max _{i, j=1,2, \ldots N, i \neq j}\left|\left(p_{i}-p_{j}\right)^{\prime} \cdot v_{1}\right|$ and $W=\max _{i, j=1,2, \ldots N, i \neq j}\left|\left(p_{i}-p_{j}\right)^{\prime} \cdot v_{3}\right|$, respectively. Figure 4 shows an example.

VS: The lesion's volume score is calculated by, $V S=N \cdot r_{x} \cdot r_{y} \cdot r_{z}$. If the volume score is smaller than 1 , we will treat it as noise and discard it.

Distance to Ostium: We also take account of the distance of the lesion to the ostium, and it is calculated by, $D=\left\|p_{o}-\bar{p}\right\|_{2}$, where $\bar{p}=\left(\sum_{i=1}^{N} p_{i}\right) / N$.

Neighborhood Relationship: A group of adjacent calcific lesions with spotty appearance are usually associated with higher event risks 10. To describe such geometric inter-dependence between the lesions, we introduce a relationship feature for each lesion, defined as: $R_{i}=\frac{1}{\sigma \sqrt{2 \pi}} \sum_{j} \exp -\left(d_{i j}\right)^{2} /\left(2 \sigma^{2}\right), j \neq i$, where the $j$-lesion is the neighbor of the $i$-lesion locating in the same artery; $\sigma$ determines the neighborhood size; $d_{i j}$ is the distance between two lesions.

Maximum HU and Average HU Values: HU value is widely to measure the density of CAC. Besides the maximum $\mathrm{HU}$ (MHU) value in the lesion, we also calculate the average $\mathrm{HU}$ (AHU) value to describe the lesion's density.

2D/3D AS: 2D AS is the popular clinical measurement used by the doctors. We also define 3D AS to measure the lesions, which is scored by the whole 3D volume and the highest $\mathrm{HU}$ value in the whole $3 \mathrm{D}$ lesion like the $2 \mathrm{D}$ AS scoring.

Artery Index: We should also indicate the artery where the lesion exists in. We label LM, LAD, LCx, and RCA by Ind $=1,2,3,4$, respectively.

\subsection{MISVM Based Cardiac Event Prediction Model}

As mentioned in the introduction, different patient's CAC scans contain different number of calcific lesions, which will result in different length of the patientscan-based feature vector. MACE is generally caused or induced by one or a few culprit or vulnerable lesions, but it is hard to label culprit or vulnerable ones without further examinations. All these issues make conventional pattern-level supervised learning methods difficult to predict cardiac event.

In this paper, we developed a novel MISVM model to predict MACE using lesion-specific CAC quantifications. MISVM is an extension of the conventional SVM classifier, in which training class labels are associated with sets of samples (or bags), instead of individual samples (or instances) 9. The labels of instances 
are only indirectly associated with the labels of bags. If a bag is labeled as positive, it means that at least one instance inside the bag is positive. A bag is labeled as negative if and only if all the instances in the bag are negative. Besides MISVM does not need the label information of instances, the size of instances in each bag can be different, so MISVM can deal with the above issues well. We take each patient as a bag, and each calcific lesion as an instance. We label the patient with MACE as a positive bag and one without MACE as a negative bag.

For convenience, we define some notations first. The patient bag $B_{i}$ has $n_{i}$ instances (lesions), $B_{i}=\left\{x_{i, 1}, x_{i, 2}, \ldots, x_{i, n_{i}}\right\}$, and its label $Y_{i}=1$ or -1 . The labels of the instances in the bag $B_{i}$ as $\left\{y_{i, j}\right\} \in\{1,-1\}_{j=1,2 \ldots, n_{i}}$, but they are unknown. $\left\{y_{i, j}\right\}$ is indirectly associated to $Y_{i}$ following this constrain:

$$
\sum_{j=1}^{n_{i}} \frac{y_{i, j}+1}{2} \geq 1: Y_{i}=1, \text { and } \forall j, y_{i, j}=-1: Y_{i}=-1 .
$$

Similar to the conventional SVM, the goal of MISVM is to find a separating hyperplane $(w, b)$ to make maximum margin between the positive instances and the negative instances, so as to efficiently separate the positive bags and the negative bags. The MISVM classifier can be defined as:

$$
\begin{gathered}
\min _{\left\{y_{i, j}\right\}} \min _{w, b, \xi} \frac{1}{2}\|w\|^{2}+C \sum_{i, j} \xi_{i, j} \\
\text { s.t. } \forall i, y_{i, j}\left(<w, x_{i, j}>+b\right) \geq 1-\xi_{i, j}, y_{i, j} \in\{1,-1\} \text { and } \xi_{i, j} \geq 0 \\
\forall i, Y_{i}=1: \sum_{j=1}^{n_{i}} \frac{y_{i, j}+1}{2} \geq 1, Y_{i}=-1: \forall j, y_{i, j}=-1
\end{gathered}
$$

where $\xi_{i, j}$ are slack factors. This is a typical mixed integer programming problem, which is difficult to be solved directly, for it aims to find the optimal labeling of instances and the optimal hyperplane based on the bags. 9 presented a heuristic optimization to solve this problem as: (1) given the labels of the instances, solve the associated quadratic program (QP) as the conventional SVM; (2)use the solution of the QP to label the instances; (3) update some labels of the instances to follow the definition of bags. The iteration continues until the labels no longer change. Initially, the labels of the instances are set according to their associated bags' labels.

\section{Experiments}

The experimental data consisted of 31 patients including 11 patients with MACE and 20 patients without MACE in 2 years after the CAC scans. $10 \mathrm{MACE}$ patients underwent invasive revascularization procedures, and another MACE patient died from a myocardium infarction. CAC examinations were performed on a 64 MDCT system (Siemens Somatom), and the images were acquired using $3 \mathrm{~mm}$ collimation with $2 \mathrm{~mm}$ inter-slice gap with non-constrast enhanced scans. Acquisition parameters included a gantry rotation of $330-375 \mathrm{~ms}$, pitch 0.24 , tube voltage $120 \mathrm{kV}$, and tube current of $250 \mathrm{mAs}$. 


\subsection{MACE Prediction Result Analysis}

We adopted three testing analysis methods to evaluate the performance of the proposed MISVM model: ROC analysis, the net reclassification improvement evaluation [11, and leave-one-out validation. We compared the proposed MISVM model with the whole AS/VS [2] and FRS [4]. Different from Cox Regression and Kaplan-Meier estimation that require longer follow-up [6/7], we focus on nearterm estimation, so we do not consider them for comparison. The Gaussian kernel is adopted for the MISVM model, $k(x, y)=\exp \left(-\gamma\|x-y\|^{2}\right)$, and $\gamma=0.65$. For two lesions in the same vessel, if their distance is less than $5 \mathrm{~mm}$, we take them as neighbors and set $\sigma=3$.

ROC Analysis: We first evaluate the proposed method by the ROC analysis. The ROC curves of the whole AS, the whole VS, and FRS were directly generated by an adaptive decision threshold search on the original values, and the ROC curve of MISVM was obtained by adaptively adjusting the decision values of SVM [12]. Figure 5 shows the comparison results, where the left is the ROC curves and the corresponding accuracy (AUC), standard error (S.E.), and $95 \%$ confidence interval (C.I.) are listed in the right. We can see MISVM obtains a better performance than the other three methods. The AUC of MISVM is 0.8318 , while the whole-heart AS/VS and FRS are $0.65,0.6636$, and 0.5075 respectively. At the sensitivity level of $80 \%$, which is commonly used as the clinical cut-off point for choosing preventive treatment, the specificity of MISVM is improved to over $75 \%$ compared to the specificities of about $35 \%$ by using the whole-heart AS/VS respectively. Because FRS is basically equal to guess from Figure 5, we will not discuss it in the following experiments.

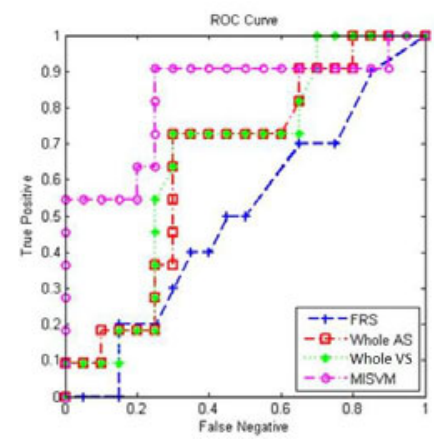

\begin{tabular}{|l|c|c|c|}
\hline & AUC & S. E. & $95 \%$ C. I. \\
\hline MISVM & 0.8318 & 0.0839 & $0.6673-0.9962$ \\
\hline Whole AS & 0.65 & 0.107 & $0.4402-0.8598$ \\
\hline Whole VS & 0.6636 & 0.1061 & $0.4557-0.8716$ \\
\hline FRS & 0.5075 & 0.1138 & $0.2844-0.7306$ \\
\hline
\end{tabular}

Fig. 5. Comparison of the ROC analysis

The Net Reclassification Improvement Evaluation: The goal of MACE risk prediction is not to simply give an individual's risk, but to help further diagnostic and therapeutic decision-making for improving clinical outcomes and lower cost. As suggested by the American Heart Association guideline, the net reclassification improvement (NRI) quantitatively assesses the improvement in risk prediction offered by the new marker by quantifying the correct movement 
Table 1. Comparison of NRI

\begin{tabular}{|l|c|c|}
\hline & $\begin{array}{c}\text { MISVM vs. } \\
\text { Whole AS }\end{array}$ & $\begin{array}{c}\text { MISVM vs. } \\
\text { Whole VS }\end{array}$ \\
\hline NRI & $76.36 \%$ & $90.45 \%$ \\
\hline$p$ & 0.001 & 0.0005 \\
\hline
\end{tabular}

Table 2. Leave-One-Out Validation

\begin{tabular}{|l|c|c|c|}
\hline & MISVM & Whole AS & Whole VS \\
\hline$\alpha_{\text {determ }}$ & $65.38 \%$ & $66.7 \%$ & $61.5 \%$ \\
\hline$\eta$ & $16.13 \%$ & $61.5 \%$ & $58.1 \%$ \\
\hline$\alpha_{\text {overall }}$ & $54.84 \%$ & $25.8 \%$ & $25.8 \%$ \\
\hline
\end{tabular}

in risk categories 13 , in which the risky is divided into three categories: a safe category that is below $90 \%$ sensitivity cut-off point for conservative treatment, a high-risk category that is above the $90 \%$ specificity cut-off point for aggressive treatment, and an indeterminate category in between the two cut-off points for further diagnostic testing. The NRI-based experimental results are reported in Table 1. NRI of MISVM over both the whole AS/VS are $76.36 \%$ with $p=$ 0.001 and $90.45 \%$ with $p=0.0005$ respectively, which shows the significant improvement of MISVM to CAC's event predication accuracy.

Leave-One-Out Validation: Furthermore, we cross validated our method on a leave-one-out basis with the above safe, high-risk, and indeterminate categories. For diagnostic purposes, physicians will be interested in improving the predictive accuracy in the safe and high-risk categories, and reducing the proportion of patients in the indeterminate category. Thus, we define three measurements for evaluation: (1). the predictive accuracy in the determinate safe and high-risk categories, $\alpha_{\text {determ }}=\left(N_{n}^{s}+N_{p}^{r}\right) /\left(N_{p}^{s}+N_{n}^{s}+N_{p}^{r}+N_{n}^{r}\right) ;(2)$. the patient percentage in the indeterminate zone, $\eta=\left(N_{t}-N_{p}^{s}-N_{n}^{s}-N_{p}^{r}-N_{n}^{r}\right) /\left(N_{t}\right) ;(3)$. the overall predictive accuracy, $\alpha_{\text {overall }}=\left(N_{n}^{s}+N_{p}^{r}\right) /\left(N_{t}\right)$, where $N_{p}^{s}$ and $N_{p}^{r}$ are the number of safe and high-risky categories in the positives respectively, $N_{n}^{s}$ and $N_{n}^{r}$ are the numbers of safe and high-risky categories in the negatives, and $N_{t}$ is the number of the total patients.

Table 2 lists the results of the leave-one-out validation. We can see that the proposed MISVM achieves similar $\alpha_{\text {determ }}$ accuracy level compared to the whole $\mathrm{AS} / \mathrm{VS}(65.38 \%, 66.7 \%$, and $61.5 \%$ respectively) in the safe and high-risky categories, while the undecidable patient percentages in the indeterminate category are largely decreased from $61.5 \%$ and $58.1 \%$ to $16.13 \%$. MISVM improves the overall predictive accuracy from $25.8 \%$ to $54.84 \%$.

\subsection{Lesion-Level Risky Analysis}

As described in Section 2.2, MISVM can also discriminate whether or not the patient has lesions of event risk. Table 3 reports the statistical mean value of safe and high-risky lesions predicted by MISVM. We can see some differences that are in good accordance with clinical observations. For instance, the highrisky lesions are statistically much larger than the safe lesions in VS, AS, length, and $\mathrm{HU}$ values. Although the statistics of the lesion distance seem inconsistent with clinical observation, we find that many small non-risky lesions with small distances to the ostia could be a reason of this inconsistency.

Additionally, we found that most high-risky lesions are from LAD. It is consistent to clinical observations: LAD is a major coronary artery that supplies 
Table 3. Overall comparison between the risky and safe lesions

\begin{tabular}{|l|c|c|c|c|c|c|c|c|}
\hline & VS & 2D AS & 3D AS & Length & Width & Distance & MHU & AHU \\
\hline Risky & 136.38 & 180.29 & 173.12 & 13.46 & 1.08 & 49.68 & 507.1 & 235.45 \\
\hline Safe & 29.37 & 36.43 & 33.18 & 5.64 & 0.81 & 28.23 & 351.5 & 196.63 \\
\hline
\end{tabular}

the most important myocardial territory. Revascularization is more likely performed in LAD than the other coronary arteries 14. For the MACE samples, we also found about $85 \%$ high-risk lesion with neighbors and $15 \%$ high-risk lesions without neighbors. Figure [6 (a) and (b) show two representative samples that were correctly predicted as MACE. The lesions in red were classified as risky, while the lesions in green were non-risky. The risky lesions predicted in (a) are relatively larger lesions with high AS and VS. The risky lesions predicted in (b) are spotty calcification. In addition, Figure 6] (c) shows a representative non-event sample that was incorrectly classified, with the red risky lesions. It is interesting to see that these lesions have both the characteristics of large size and spotty calcification, which suggests that CAC scan might not be a complete indicator of MACE. In future work, we plan to include more clinical cues, such as patients' age, gender, history of smoking, hypertension, and diabetics, and biomarkers from blood tests, to develop a more accurate event predictor.

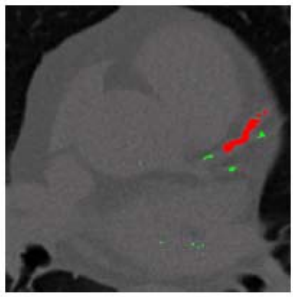

(a)

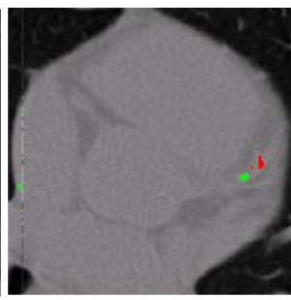

(b)

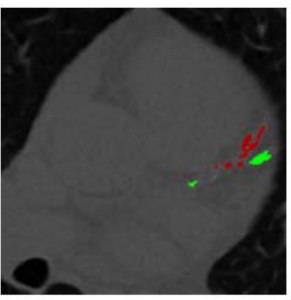

(c)

Fig. 6. Representative samples. (a) and (b) are two positive samples that are correctly classified; (c) is a negative sample that is incorrectly classified as positive.

\section{Conclusion}

In this paper, we proposed a MISVM based lesion-specific CAC quantification framework to improve the event prediction value of CAC scanning. Different from the conventional whole-heart CAC scores, it took account of a more comprehensive lesion-specific CAC information from routine CAC scans, and achieved better prediction sensitivity and specificity. Besides event prediction, it can also be used to characterize culprit or vulnerable calcific lesions, and evaluate their associated event risks. Experimental results demonstrated that it has the potential to significantly improve the clinical predictive value of CAC. 


\section{References}

1. Detrano, R., et al.: Coronary calcium does not accurately predict near-term future coronary events in high-risk adults. Circulation 99(20), 2633-2638 (1999)

2. Agatston, A., Janowitz, W., et al.: Quantification of coronary artery calcium using ultrafast computed tomography. J. Am. Coll. Cardiol. 15(4), 827-832 (1990)

3. Callister, T., Cooil, B., Raya, S., et al.: Coronary artery disease: improved reproducibility of calcium scoring with an electron-beam ct volumetric method. Radiology 208(3), 807-814 (1998)

4. Greenland, P., LaBree, L., Azen, S., et al.: Coronary artery calcium score combined with framingham score for risk prediction in asymptomatic individuals. Journal of the American Medical Association 291, 210-215 (2004)

5. Brown, E., Kronmal, R., et al.: Coronary calcium coverage score: determination, correlates, and predictive accuracy in the multi-ethnic study of atherosclerosis. Radiology 247(3), 669-675 (2008)

6. Lakoski, S., et al.: Coronary artery calcium scores and risk for cardiovascular events in women classified as "low risk" based on framingham risk score: The multi-ethnic study of atherosclerosis. Arch. Intern. Med. 167(22), 2437-2442 (2007)

7. Greenland, P., et al.: Coronary artery calcium score combined with framingham score for risk prediction in asymptomatic individuals. JAMA 291, 210-215 (2004)

8. Qian, Z., Marvasty, I., et al.: Lesion-specific coronary artery calcium quantification better predicts cardiac events. In: Int'l. Symposium on Biomedical Imaging (2009)

9. Andrews, R., Tsochantaridis, I., Hofmann, T.: Support vector machines for multiple instance learning. In: Advances in Neural Information Processing Systems (2003)

10. Ehara, S., Kobayashi, Y., et al.: Spotty calcification typifies the culprit plaque in patients with acute myocardial infarction: an intravascular ultrasound study. Circulation 110(22), 3424-3429 (2004)

11. Pencina, M., D'Agostino, S.R., et al.: Evaluating the added predictive ability of a new marker: From area under the roc curve to reclassification and beyond. Sta. Med. 27(2), 157-172 (2008)

12. Fan, R.E., Chen, P.H., Lin, C.J.: Working set selection using the second order information for traing svm. J. Machine Learning Research 6, 1889-1918 (2005)

13. Hlatky, M., Greenland, P., et al.: Criteria for evaluation of novel markers of cardiovascular risk: A scientific statement from the american heart association. Circulation 119(17), 2408-2416 (2009)

14. Virmani, R., Burke, A., et al.: Pathology of the vulnerable plaque. J. AM Coll. Cardiol. 47(8), C13-C18 (2006) 